J. Phys. IV France 1 (2018)

$\operatorname{Pr} 1-1$

(c) EDP Sciences, Les Ulis

\title{
Charge stripes in cuprate superconductors: The middle way
}

\author{
J. M. Tranquada ${ }^{1}$ \\ ' ${ }^{1}$ Brookhaven National Laboratory, Upton, NY 11973-5000, USA
}

\begin{abstract}
Charge and spin stripe order is a type of electronic crystal observed in certain layered cuprates associated with high-temperature superconductivity. Quantum-disordered stripes could be relevant for understanding the superconductivity. Here I discuss recent experimental characterizations of the stripe-ordered state in $\mathrm{La}_{1.875} \mathrm{Ba}_{0.125} \mathrm{CuO}_{4}$, and compare them with properties of superconducting compositions.
\end{abstract}

\section{INTRODUCTION}

For two-dimensional (2D) electronic systems, there are two limiting ground states. When kinetic energy dominates, one obtains a Fermi liquid, with delocalized quasiparticles. On ' the other hand, when Coulomb repulsion dominates, one ends up with electrons localized in a Wigner crystal [1]. The parent compounds of cuprate superconductors are Mott-Hubbard charge-transfer insulators, where neither of the limiting ground states is applicable. The doped antiferromagnetic insulator becomes metallic and superconducting, with a normal state that is not well understood. There have been attempts to describe various aspects of doped cuprates starting with either a Fermi-liquid picture [2] or a Wigner crystal description [3-5], though there are features of each that are not entirely satisfactory.

As discussed at this workshop by Boris Spivak, there is also a "middle way". A proper treatment of the transition from a Fermi liquid state to a Wigner crystal indicates that there should be intermediate "micro-emulsion" phases [6]. In the case of hole-doped antiferromagnets such as cuprates, these are predicted to be stripe phases [7-10]. Indeed, ordered charge and spin stripes have been observed in certain cuprates (especially near a hole concentration of $\frac{1}{8}$ per $\mathrm{Cu}$ site) $[11,12]$ and in layered nickelates over a broad range of doping [13]. Static stripe order competes with superconductivity [11], but quantum-disordered stripes could be compatible, and perhaps responsible for, high-temperature superconductivity [14,15].

In this paper, I will discuss some recent results for $\mathrm{La}_{1.875} \mathrm{Ba}_{0.125} \mathrm{CuO}_{4}$, a compound that exhibits static stripe order at temperatures below $\approx 50 \mathrm{~K}[12]$. The magnetic excitation spectrum in the ordered state [16] looks very much like that in superconducting $\mathrm{YBa}_{2} \mathrm{Cu}_{3} \mathrm{O}_{6+x}$ [17]. New measurements on $\mathrm{La}_{1.875} \mathrm{Ba}_{0.125} \mathrm{CuO}_{4}$ in the paramagnetic state indicate that the magnetic excitations change relatively little, consistent with maintaining fluctuating stripe correlations [18]. New optical conductivity [19] and photoemission measurements [20] on the same material indicate that stripes are compatible with the so-called nodal-metal state, but also suggest a charge-density-wave gap within the stripes that is presumably responsible for the suppression of superconductivity [21]. This leads to a new view of stripes and metallic transport. To appreciate some of these results, I will first discuss some characteristics of the "pseudogap" phase in the next section. 


\section{CHARACTERISTICS OF THE PSEUDOGAP PHASE}

It is common to describe the normal state of underdoped cuprate superconductors as a pseudogap phase, at least for temperatures below some crosssover $T^{*}$ [22]. There are two types of features associated with the pseudogap concept. One is the general depression of the density of states near the Fermi level, $E_{\mathrm{F}}$, in underdoped cuprates compared to what one might expect for weakly interacting electrons. A second is the temperature-dependent depression of signal seen first in optical conductivity measured with the polarization along the $c$-axis (perpendicular to the $\mathrm{CuO}_{2}$ planes) [23] and soon after in angle-resolved photoemission (ARPES) measurements in the vicinity of $(\pi, 0)$ (the "antinodal" region) $[24,25]$. That the depressed density of states, on the one hand, and the temperature-dependent effects, on the other, are distinct phenomena becomes very clear when one considers studies of the optical conductivity parallel to the $\mathrm{CuO}_{2}$ planes, $\sigma_{a b}$.

Undoped, insulating cuprates have a charge transfer gap of 1.5 to $2 \mathrm{meV}$. As shown by Uchida et al. [26] for the case of $\mathrm{La}_{2-x} \mathrm{Sr}_{x} \mathrm{CuO}_{4}$, hole doping introduces finite conductivity into the gap, and this conductivity grows with doping up to $x \sim 0.2$. (The growth in integrated conductivity is opposite to what one would expect if the conduction electrons were noninteracting.) This effect is visible at room temperature; on cooling, $\sigma_{a b}$ shows no loss of density of states in the normal state [27]. (Below the superconducting transition temperature, $T_{c}$, the contribution from electrons that participate in the superfluid move to zero frequency, leaving a gap-like feature in $\sigma_{a b}$.)

In contrast to the 1-eV energy scale for the "depressed" density of states, the temperaturedependent pseudogaps seen in ARPES and $\sigma_{c}$ have an energy scale on the order of $50 \mathrm{meV}$, comparable to the the superconducting gap maximum. Clearly, the temperature-dependent effect occurs on an energy scale that is much smaller than that associated with the general depression of the density of states.

While no temperature-dependent pseudogaps are seen in $\sigma_{a b}$, there is nevertheless an interesting variation in $\sigma_{a b}(\omega)$ as $T$ is reduced towards $T_{c}$. At high temperatures, the conductivity of underdoped cuprates is rather flat as a function of frequency $[28,29]$. On cooling, the conductivity below $\sim 0.1 \mathrm{eV}$ narrows into a Drude peak, while that at higher energies changes little. At the lower temperatures it becomes possible to decompose the spectrum into two components: a narrow Drude peak at low frequency and a broad Lorentzian oscillator (the "mid-IR" peak) with a doping-dependent mid-point in the range $0.2-0.5 \mathrm{eV}$ [29-31]. In the superconducting state, the superfluid density comes almost entirely from the Drude peak [29]. It should be noted that the integrated conductivity in the Drude peak is only a quarter of that in the mid-IR $[29,30]$.

The Drude peak has to come from states close to $E_{\mathrm{F}}$. In the underdoped cuprates, these states are along the Fermi arc [25,32], centered on the nodal point [the point at which the $d$-symmetry superconducting gap goes to zero, close to $\left.\left(\frac{\pi}{2}, \frac{\pi}{2}\right)\right]$. The regime, below $T^{*}$, where the Drude peak can be resolved has been called a nodal-metal state [29]. I believe that this is a more useful description than the more ambiguous "pseudogap" phase.

It was originally noted by Ido et al. [33] that the energy, magnitude, and doping dependence of the mid-IR peak in $\mathrm{La}_{2-x} \mathrm{Sr}_{x} \mathrm{CuO}_{4}$ is similar to that in the nickelates, $\mathrm{La}_{2-x} \mathrm{Sr}_{x} \mathrm{NiO}_{4}$. It was only later that the existence of diagonal stripe order was identified in the nickelates [34]. An interpretation of the mid-IR peak in the nickelates in terms of the electronic structure of stripes has recently been proposed [35]. The main difference between the cuprates and nickelates is the absence of a Drude component in the latter. It should be noted, however, that a Drude peak has been observed in the cuprate $\mathrm{La}_{1.275} \mathrm{Nd}_{0.6} \mathrm{Sr}_{0.125} \mathrm{CuO}_{4}$ [36], which should exhibit stripe order as in similar compositions with slightly less Nd [11]. Lorenzana and Seibold [37] have 


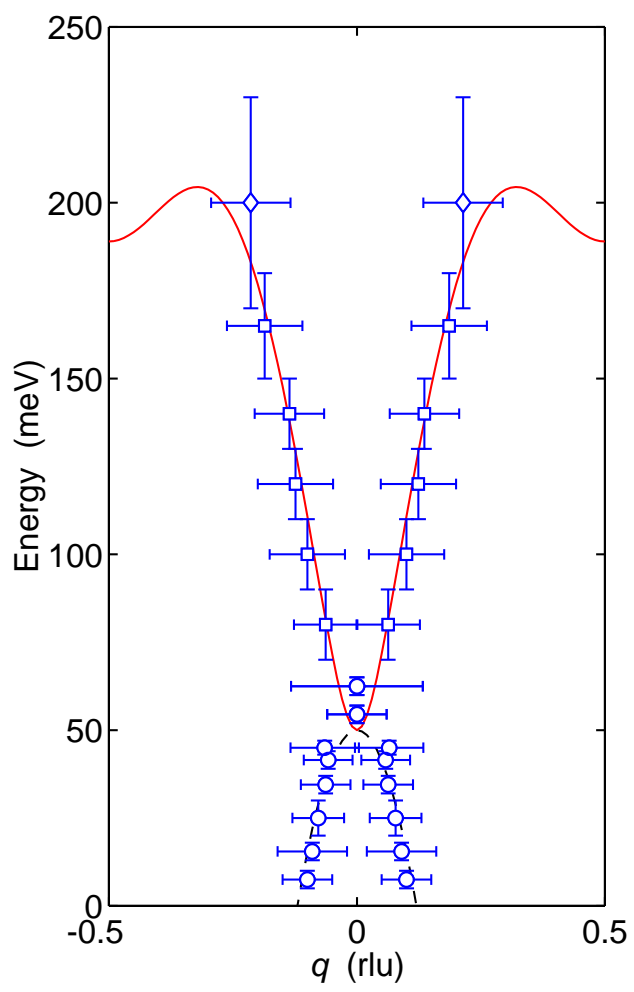

Fig. 1. Symbols: experimentally measured dispersion [16] of magnetic excitations along $\mathbf{Q}=(0.5+$ $q, 0.5, l)$ in stripe-ordered $\mathrm{La}_{1.875} \mathrm{Ba}_{0.125} \mathrm{CuO}_{4}$. Horizontal bars indicate the fitted half-width of the scattering. The solid line is the calculated [39] dispersion along a two-leg ladder with a superexchange energy of $J=100 \mathrm{meV}$.

obtained both Drude and mid-IR contributions in a calculation for cuprate stripes.

\section{UNIVERSAL MAGNETIC EXCITATION SPECTRUM}

As mentioned before, $\mathrm{La}_{1.875} \mathrm{Ba}_{0.125} \mathrm{CuO}_{4}$ exhibits static charge and spin stripe order for $T<$ $50 \mathrm{~K}$. The magnetic excitation has been measured at $T=12 \mathrm{~K}$ using the MAPS spectrometer at the ISIS Facility [16]. The dispersion along a line through the positions of a pair of magnetic superlattice peaks is shown in Fig. 1. At low energies, the excitations disperse upwards out of the superlattice peaks as expected for spin waves [12]. With increasing energy, we would expect to begin to resolve cones of spin waves, as observed in stripe-ordered $\mathrm{La}_{2-x} \mathrm{Sr}_{x} \mathrm{NiO}_{4}$ [38]; in contrast, we can only identify inwardly dispersing excitations that merge at about $50 \mathrm{meV}$. Above that energy the excitations disperse outward again, forming an hour-glass shape.

While the observed dispersion differs from the simplest spin-wave predictions, it turns out to be quite similar to what is observed in superconducting $\mathrm{YBa}_{2} \mathrm{Cu}_{3} \mathrm{O}_{6+x}[17,40-44]$ and $\mathrm{La}_{2-x} \mathrm{Sr}_{x} \mathrm{CuO}_{4}$ [45]. The main difference among these systems is that there is a spin gap in the superconducting state [46], with a pile up of weight above the spin gap [45, 47]. The magnitude of the spin gap is roughly proportional to $T_{c}$ [48].

Guangyong $\mathrm{Xu}$ has recently extended the magnetic excitation measurements for 
$\mathrm{La}_{1.875} \mathrm{Ba}_{0.125} \mathrm{CuO}_{4}$ to temperatures above the stripe-ordering transition [18]. At $65 \mathrm{~K}$, just above the transition, the main differences are at energies below $10 \mathrm{meV}[12,18]$, and are of the type expected in a magnetic system that goes through a disordering transition. At higher energies, the main effect seems to be a broadening of the spectrum in terms of momentum widths. The general features of the spectrum are still recognizable at $300 \mathrm{~K}$. The smooth evolution of the magnetic spectrum suggests that the high-temperature state is a dynamically-disordered version of the stripe-ordered phase; in other words, we have evidence for a fluctuating stripe phase. The similarity of the spectrum with that of $\mathrm{YBa}_{2} \mathrm{Cu}_{3} \mathrm{O}_{6+x}$ and $\mathrm{La}_{2-x} \mathrm{Sr}_{x} \mathrm{CuO}_{4}$ then suggests that quantum-disordered stripes may be common to the superconducting cuprates.

\section{ELECTRONIC NATURE OF THE STRIPE-ORDERED STATE}

A remaining question is: are the electronic properties of a static or fluctuating stripe state compatible with the nodal-metal phase discussed earlier? As a first test, Dordevic and Homes [19] have measured the in-plane optical conductivity on a cleaved crystal of $\mathrm{La}_{1.875} \mathrm{Ba}_{0.125} \mathrm{CuO}_{4}$. The results are generally quite similar to those in the normal state of $\mathrm{La}_{2-x} \mathrm{Sr}_{x} \mathrm{CuO}_{4}$ with $x=0.125$ [36]. In particular, the conductivity below $50 \mathrm{~cm}^{-1}(\sim 6 \mathrm{meV})$ grows with cooling, even in the stripe-ordered state. Those low-energy excitations must be associated with the nodal states along the Fermi arc [32]. A new behavior is the gap-like loss of weight from the range $150-300 \mathrm{~cm}^{-1}$ on cooling into the stripe-ordered phase. This loss of weight is likely to occur in the antinodal region of reciprocal space.

Tonica Valla, working with Alexei Federov, has very recently succeeded in measuring angleresolved photoemission from $\mathrm{La}_{1.875} \mathrm{Ba}_{0.125} \mathrm{CuO}_{4}$ [20]. Although the measurements were obtained with an energy resolution of $30-35 \mathrm{meV}$, they provide clear evidence for a Fermi arc and nodal quasiparticles. This indicates that stripes are compatible with the nodal metal; in fact, the main conduction path is at $45^{\circ}$ to the stripes.

The higher-energy gap-like behavior seen in the optical conductivity suggests that there might be a charge-density wave (CDW) along the stripes in the ordered phase. The competition between CDW and superconducting correlations within the stripes has been discussed by Kivelson, Fradkin, and Emery [21], and the nesting of Fermi-surface segments associated with stripes was noted by Zhou et al. [49], with $2 k_{\mathrm{F}}=a^{*} / 4$. The CDW would require electronphonon coupling, and hence one might expect to see a phonon anomaly at $\mathbf{q}=\left(2 k_{\mathrm{F}}, 0,0\right)$ (for stripes running along the $x$ direction). Intriguingly, Reznik et al. [50] have observed a 10-15 meV energy broadening of the longitudinal-optical bond-stretching branch at just this wave vector. (Because of the nature of the crystal structure, the measurement averages over directions parallel and perpendicular to the stripes, so that it is not possible to tell directly in which orientation the anomaly occurs.) Further measurements are in progress.

\section{NEW VIEW OF STRIPES AND METALLIC TRANSPORT}

The new experimental results are changing the picture of metallic transport in a striped phase. Previously, the simplest assumption was that charge would move more easily along a stripe than perpendicular to it. The optical conductivity [19] and photoemission [20] results now indicate that the best metallic conduction is at $45^{\circ}$ to the stripes. It appears, then, that stripe correlations are compatible with the nodal-metal state. It remains a challenge for theory to explain these results.

I would like to thank my many experimental collaborators, including S. Dordevic M. Fujita, G. D. Gu, C. C. Homes, M. Hücker, L. Pintschovius, D. Reznik, T. Valla, G. Xu, and K. Yamada. Research at Brookhaven is 
supported by the U.S. Department of Energy's Office of Science under Contract No. DE-AC02-98CH10886.

\section{References}

[1] D. Baeriswyl and S. Fratini, in this volume; cond-mat/0509112

[2] A. V. Chubukov, D. Pines, and J. Schmalian, in The Physics of Superconductors Vol I: Conventional-, High-Transition Temperature, and Novel Superconductors, edited by K. H. Bennemann and J. B. Ketterson (Springer-Verlag, Berlin, 2003), pp. 495-590.

[3] M. Franz, Science 305, 1410 (2004).

[4] G. Rastelli, S. Fratini, and P. Quémerais, Eur. Phys. J. B 42, 305 (2004).

[5] S. Komiya, H.-D. Chen, S.-C. Zhang, and Y. Ando, Phys. Rev. Lett. 94, 207004 (2005).

[6] R. Jamei, S. Kivelson, and B. Spivak, Phys. Rev. Lett. 94, 056805 (2005).

[7] S. A. Kivelson, I. P. Bindloss, E. Fradkin, V. Oganesyan, J. M. Tranquada, A. Kapitulnik, and C. Howald, Rev. Mod. Phys. 75, 1201 (2003).

[8] J. Zaanen, O. Y. Osman, H. V. Kruis, Z. Nussinov, and J. Tworzydło, Phil. Mag. B 81, 1485 (2001).

[9] K. Machida, Physica C 158, 192 (1989).

[10] S. Sachdev and N. Read, Int. J. Mod. Phys. B 5, 219 (1991).

[11] N. Ichikawa, S. Uchida, J. M. Tranquada, T. Niemöller, P. M. Gehring, S.-H. Lee, and J. R. Schneider, Phys. Rev. Lett. 85, 1738 (2000).

[12] M. Fujita, H. Goka, K. Yamada, J. M. Tranquada, and L. P. Regnault, Phys. Rev. B 70, 104517 (2004).

[13] H. Yoshizawa, T. Kakeshita, R. Kajimoto, T. Tanabe, T. Katsufuji, and Y. Tokura, Phys. Rev. B 61, R854 (2000).

[14] V. J. Emery, S. A. Kivelson, and O. Zachar, Phys. Rev. B 56, 6120 (1997).

[15] E. Arrigoni, E. Fradkin, and S. A. Kivelson, Phys. Rev. B 69, 214519 (2004).

[16] J. M. Tranquada, H. Woo, T. G. Perring, H. Goka, G. D. Gu, G. Xu, M. Fujita, and K. Yamada, Nature 429, 534 (2004).

[17] S. M. Hayden, H. A. Mook, P. Dai, T. G. Perring, and F. Doğan, Nature 429, 531 (2004).

[18] G. Xu, T. G. Perring, G. D. Gu, M. Fujita, K. Yamada, and J. M. Tranquada, (unpublished).

[19] S. V. Dordevic, C. C. Homes, and G. D. Gu, (unpublished).

[20] T. Valla, A. Federov, and G. D. Gu, (unpublished).

[21] S. A. Kivelson, E. Fradkin, and V. J. Emery, Nature 393, 550 (1998).

[22] T. Timusk and B. Statt, Rep. Prog. Phys. 62, 61 (1999).

[23] C. C. Homes, M. Reedyk, D. Crandles, and T. Timusk, Appl. Opt. 32, 2972 (1993).

[24] A. G. Loeser, Z. X. Shen, D. S. Dessau, D. S. Marshall, C. H. Park, P. Fournier, and A. Kapitulnik, Science 273, 325 (1996).

[25] M. R. Norman, H. Ding, M. Randeria, J. C. Campuzano, T. Yokoya, T. Takeuchi, T. Takahashi, T. Mochiku, K. Kadowaki, P. Guptasarma, and D. G. Hinks, Nature 392, 157 (1998).

[26] S. Uchida, T. Ido, H. Takagi, T. Arima, and Y. Tokura, Phys. Rev. B 43, 7942 (1991).

[27] A. F. Santander-Syro, R. P. S. M. Lobo, N. Bontemps, W. Lopera, D. Giratá, Z. Konstantinovic, Z. Z. Li, and H. Raffy, Phys. Rev. B 70, 134504 (2004).

[28] K. Takenaka, J. Nohara, R. Shiozaki, and S. Sugai, Phys. Rev. B 68, 134501 (2003).

[29] Y. S. Lee, K. Segawa, Z. Q. Li, W. J. Padilla, M. Dumm, S. V. Dordevic, C. C. Homes, Y. Ando, and D. N. Basov, Phys. Rev. B 72, 054529 (2005).

[30] W. J. Padilla, Y. S. Lee, M. Dumm, G. Blumberg, S. Ono, K. Segawa, S. Komiya, Y. Ando, and D. N. Basov, Phys. Rev. B 72, 060511(R) (2005).

[31] D. B. Romero, C. D. Porter, D. B. Tanner, L. Forro, D. Mandrus, L. Mihaly, G. L. Carr, and G. P. Williams, Phys. Rev. Lett. 68, 1590 (1992).

[32] T. Yoshida, X. J. Zhou, T. Sasagawa, W. L. Yang, P. V. Bogdanov, A. Lanzara, Z. Hussain, T. Mizokawa, A. Fujimori, H. Eisaki, Z.-X. Shen, T. Kaneshita, and S. Uchida, Phys. Rev. Lett. 91, 027001 (2003).

[33] T. Ido, K. Magoshi, H. Eisaki, and S. Uchida, Phys. Rev. B 44, 12094 (1991).

[34] J. M. Tranquada, D. J. Buttrey, V. Sachan, and J. E. Lorenzo, Phys. Rev. Lett. 73, 1003 (1994).

[35] C. C. Homes, J. M. Tranquada, Q. Li, A. R. Moodenbaugh, and D. J. Buttrey, Phys. Rev. B 67, 184516 (2003).

[36] M. Dumm, D. N. Basov, S. Komiya, Y. Abe, and Y. Ando, Phys. Rev. Lett. 88, 147003 (2002). 
[37] J. Lorenzana and G. Seibold, Phys. Rev. Lett. 90, 066404 (2003).

[38] H. Woo, A. T. Boothroyd, K. Nakajima, T. G. Perring, C. D. Frost, P. G. Freeman, D. Prabhakaran, K. Yamada, and J. M. Tranquada, Phys. Rev. B 72, 064437 (2005).

[39] T. Barnes and J. Riera, Phys. Rev. B 50, 6817 (1994).

[40] M. Arai, T. Nishijima, Y. Endoh, T. Egami, S. Tajima, K. Tomimoto, Y. Shiohara, M. Takahashi, A. Garrett, and S. M. Bennington, Phys. Rev. Lett. 83, 608 (1999).

[41] P. Bourges, Y. Sidis, H. F. Fong, L. P. Regnault, J. Bossy, A. Ivanov, and B. Keimer, Science 288, 1234 (2000).

[42] D. Reznik, P. Bourges, L. Pintschovius, Y. Endoh, Y. Sidis, T. Matsui, and S. Tajima, Phys. Rev. Lett. 93, 207003 (2004).

[43] C. Stock, W. J. L. Buyers, R. Liang, D. Peets, Z. Tun, D. Bonn, W. N. Hardy, and R. J. Birgeneau, Phys. Rev. B 69, 014502 (2004).

[44] S. Pailhès, Y. Sidis, P. Bourges, V. Hinkov, A. Ivanov, C. Ulrich, L. P. Regnault, and B. Keimer, Phys. Rev. Lett. 93, 167001 (2004).

[45] N. B. Christensen, D. F. McMorrow, H. M. Rønnow, B. Lake, S. M. Hayden, G. Aeppli, T. G. Perring, M. Mangkorntong, M. Nohara, and H. Tagaki, Phys. Rev. Lett. 93, 147002 (2004).

[46] J. M. Tranquada, H. Woo, T. G. Perring, H. Goka, G. D. Gu, G. Xu, M. Fujita, and K. Yamada, cond-mat/0411082

[47] J. M. Tranquada, C. H. Lee, K. Yamada, Y. S. Lee, L. P. Regnault, and H. M. Rønnow, Phys. Rev. B 69 , 174507 (2004).

[48] J. M. Tranquada, cond-mat/0508272

[49] X. J. Zhou, P. Bogdanov, S. A. Kellar, T. Noda, H. Eisaki, S. Uchida, Z. Hussain, and Z.-X. Shen, Science 286, 268 (1999).

[50] D. Reznik, L. Pintschovius, M. Ito, S. Iikubo, M. Sato, H. Goka, M. Fujita, K. Yamada, G. D. Gu, and J. M. Tranquada, (unpublished). 